\title{
Incidence and Etiology of Catheter Associated Urinary Tract Infection among Admitted Patients at Kabale Regional Referral Hospital, South Western Uganda
}

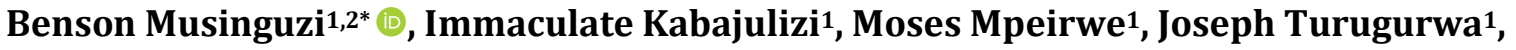 \\ Taseera Kabanda ${ }^{1}$ \\ ${ }^{1}$ Department of Microbiology, Faculty of Medicine, Mbarara University of Science and Technology, Mbarara, Uganda \\ ${ }^{2}$ Department of Medical Laboratory Sciences, School of Allied Health Sciences, Kampala International University, Bushenyi, Uganda \\ Email:*musinguzi.benson@kiu.ac.ug
}

How to cite this paper: Musinguzi, B., Kabajulizi, I., Mpeirwe, M., Turugurwa, J. and Kabanda, T. (2019) Incidence and Etiology of Catheter Associated Urinary Tract Infection among Admitted Patients at Kabale Regional Referral Hospital, South Western Uganda. Advances in Infectious Diseases, 9, 183-196.

https://doi.org/10.4236/aid.2019.93014

Received: June 19, 2019

Accepted: July 21, 2019

Published: July 24, 2019

Copyright $\odot 2019$ by author(s) and ScientificResearch Publishing Inc.

This work is licensed under the CreativeCommons Attribution International License (CC BY 4.0).

http://creativecommons.org/licenses/by/4.0/ (c) (i) Open Access

\begin{abstract}
Introduction: Catheter Associated Urinary Tract Infection is the most common hospital acquired infection worldwide. Urinary Tract Infections among catheterised patients are on rise regardless of antibiotic use and this is due to erratic use of antibiotics, treatment failure, antimicrobial resistance and emergency of Extended Spectrum Beta Lactamase producing bacteria leading to patient distress, increased healthcare costs, long hospital stay and poor patient response to antibiotics. In Uganda, no previous studies have sought to study the burden of CAUTI among catheterized patients, the bacterial pathogens involved and their antimicrobial susceptibility patterns yet there is upsurge in antimicrobial resistance of uropathogens. The effective management of patients suffering from Catheter Associated Urinary Tract infection (CAUTI) relays on the identification of uropathogens that cause CAUTI and the selection of an effective antibiotic agent to the uropathen in question. Objectives: The objectives of this study were to determine incidence, etiology and antibiotic susceptibility pattern among the uropathogens causing Catheter Associated Urinary Tract Infections among patients with indwelling catheters at Kabale Regional Referral Hospital. Methods: Using a descriptive prospective observational hospital-based study, the study was conducted on $150 \mathrm{ca}-$ theterized patients recruited from Emergency, Obstetrics and gynecology, Medical, Maternity and Surgical wards at Kabale Regional Referral Hospital between April and May 2019. The urine samples from study participants were processed in Kabale RRH microbiology laboratory as per standard operating procedures. After isolation and identification, all the isolates were subjected
\end{abstract}


to antibiotic susceptibility testing for commonly used antibiotics. Results: Following the urine culture from 150 catheterized patients, urine from 23 (15.3\%) patients showed significant growth. The common bacterial isolates were Escherichia coli 12 (52\%), followed by the Klebsiella pneumoniae 6 (26\%), Staphylococcus aureus 3 (13\%) and Pseudomonas spp. 2 (8.7\%). All Gram-negative isolates were sensitive to Imipenem 20 (100\%) while all $S$. aureus isolates (3) were $100 \%$ sensitive to Vancomycin and Cefoxitin. Isolates were sensitive to Gentamicin 20 (82.6), Ceftriaxone 16 (69.6), Ciprofloxacin 10 (43.5) and Nitrofurantoin 9 (39.1). All isolates were $100 \%$ resistant to Cotrimoxazole. 6 gram negative isolates were resistant to ceftazidime and were tested for Extended Spectrum Beta (B) Lactamase (ESBL), 5 (83.3\%) were identified as ESBL-producing bacteria. $K$. pneumonia $3(60 \%)$ presented the highest percentage of ESBLs as compared to E. coli 2 (40\%). Conclusions: The Incidence of CAUTI among patients with indwelling urinary catheters at Kabale Regional Referral Hospital is high (15.3\%) and is mostly caused by $E$. coli and $K$. pneumoniae. These bacteria are resistant to most commonly used antibiotics and thus there is a need to put more emphasis on CAUTI prevention strategies and use culture and sensitivity tests before prescription of antibiotics.

\section{Keywords}

Catheter Associated Urinary Tract Infection(CAUTI), ESBL, Uropathogens, Urinary Tract Infection, Incidence

\section{Introduction}

Catheter associated urinary tract infection (CAUTI) is the commonest hospital associated Urinary tract infection worldwide with an incidence of approximately $35 \%$ [1] and is one of the leading causes of morbidity and mortality among admitted patients with indwelling urinary catheters [2]. Over one hundred and fifty (150) million people are affected per annum costing more than 6 billion US dollars [3]. Catheter associated urinary tract infections occur with very high incidence of about $20 \%$ once catheterized patients are maintained on closed drainage [4].

It is as a result of bacteria being introduced into the urethra during insertion of the catheter through the sheath of exudates surrounding the catheter or intraluminally from catheter collection bag or the tube [5] and is defined as bacteriuria with a count of $\geq 10^{5} \mathrm{CFUs} / \mathrm{ml}$ [6].

More than 25\% of patients catheterised for a duration of more than 7 days develop bacteriuria [5] and this increases on admission duration and treatment costs as well [7].

Urinary catheter is seldomly colonised by pathogenic bacteria like Escherichia coli, Klebsiella, Enterococci, Pseudomonas, Enterobacter, Proteus and Serratia whose pathogenicity is added by biofilm formation which protects the organism 
from body's innate defences. The source of bacteria causing CAUTI can be exogenous via contaminated hands of healthcare workers or equipment or endogenous via rectal, meatal or vaginal colonization moreover many of these bacteria are part of patients endogenous bowel flora, but they can be acquired from the hospital as well [2].

The effects of CAUTI are devastating to admit patients and approximately $10 \%-15 \%$ of such patients with indwelling catheters die per year [8]. Other adverse effects include the emergence of resistant bacteria due to inappropriate antibiotics prescription, stricture formation due to inflammation of surrounding tissues, purulent urethritis, prostate gland abscesses, and inflammation of the prostate gland [9] yet their use is inevitable especially in bladder drainage prior to, during, or after surgery, relieving urine retention, measuring the urine output and relieving urine incontinence [10].

The risk of getting CAUTI depends on host susceptibility, quality of catheter care, method and duration of catheterization. Host factors including female gender, severe underlying illness, prolonged catheterization, disconnection of the catheter and drainage tube and lack of systemic antimicrobial prophylaxis which increase the possibility of CAUTI [2].

60 to 80 percent of the patients with CAUTI receive inappropriate antibiotics and this has led to emergence of resistant organisms such as extended spectrum beta-lactamase producing Enterobacteriaceae and multidrug resistant E. coli [6]. There is a growing concern by Kabale Regional Referral Hospital clinical team about increased catheter related complications and high ESBL producing uropathogens which is a big challenge during patient management [11].

This study aimed at determining the incidence, etiology and antibiotic susceptibility pattern among the uropathogens causing Catheter Associated Urinary Tract Infections among admitted patients with indwelling catheters at Kabale Regional Referral Hospital.

\section{Materials and Methods}

\subsection{Study Design}

This was a descriptive prospective observational hospital-based study, the study was conducted on 150 catheterized patients selected by convenience sampling from Emergency, Obstetrics and gynecology, Medical, Maternity and Surgical wards at Kabale Regional Referral Hospital (KRRH) in western Uganda between April and May 2019. Selected and eligible participants were followed up for a maximum of 5 days. The follow up urine samples were collected on the 3rd and 5th day following catheterization.

\subsection{Inclusion Criteria}

1) Admitted patients with indwelling urinary catheters who signed screening consent forms to allow their urine samples to be screened for urinary tract infections within 1 hour of urethral catheterisation and are found to be urine culture 
negative.

2) Patients who were urine culture negative immediately after catheterisation and either signed informed consent forms for conscious adult patients or surrogate consent forms for unconscious adults who are unable to give informed consent or minor assent forms for case of children below 18years after seeking consent from parents/guardians.

\subsection{Exclusion Criteria}

1) Patients with a positive baseline urine culture;

2) Patients discharged before the 3rd day ( 48 hours) of catheterisation;

3) Patients in whom the catheter have been removed before the 3rd day ( 48 hours) of catheterisation.

\subsection{Sample Collection and Transport}

Open technique was used to collect urine samples from the catheter aseptically into a sterile urine container and transported to the Kabale RRH microbiology laboratory for analysis within 30 minutes. The samples were processed as per standard laboratory standard operating procedures. Urine analysis, culture and antibiotic susceptibility testing were tests done.

\subsection{Urinalysis}

Baseline urine dipstick test was performed by dipping the strips into the urine, and interpreted according to the instructions of the manufacturer to detect leukocyte esterase (LE) activity as an indicator of pyuria and urinary nitrite (NIT) production, the indicators of bacteriuria. Urine Microscopy was done to detect and quantify pus cells.

\subsection{Isolation and Identification of Microorganisms}

For primary Isolation, $0.01 \mathrm{ml}$ of the urine specimen was inoculated on to Cystine-Lactose-Electrolyte Deficient (CLED) medium and Blood agar using calibrated sterile wire loop. The inoculated plates were incubated at $37^{\circ} \mathrm{C}$ for 24 hours and observed for growth and in case of no growth, the plate was incubated up to 48 hours before declaring absence of bacterial growth. Bacterial growth was identified to determine the microorganisms involved in CAUTI. Urine samples with colony $\geq 10^{5} \mathrm{Cfu} / \mathrm{ml}$ were taken as significant growth positive urine culture) [12]. Isolates were identified on the basis of colony morphology, haemolytic pattern, gram reactions, microscopic examination and biochemical tests.

\subsection{Bacterial Antibiotic Susceptibility Testing}

Antibiotic susceptibility test was done on pure colonies of each species to commonly used antibiotics using the disc diffusion method and using the Kirby Bauer disk diffusion technique with commercially available disks on Mueller Hinton agar 
plates. The viability of antibiotics disks were quality controlled weekly using $E$. coli ATCC 25922 and Staphylococcus aureus ATCC 25923 as recommended by the clinical laboratory standards institute (CSLI) \{Patel, 2017\}. The following antibiotics were used: Gentamicin $(10 \mu \mathrm{g})$, ceftriazone $(30 \mu \mathrm{g})$, Ciprofloxacin $(5 \mu \mathrm{g})$, Nitrofurantion $(10 \mu \mathrm{g})$, Cefoxitin disc $(30 \mu \mathrm{g})$, Imipenem $(10 \mu \mathrm{g})$, Ceftazidime (30 $\mu \mathrm{g})$, Ceftazidime-clavulanic acid $(30 / 10 \mu \mathrm{g})$, Cotrimoxazole (trimethoprim + sulfamethoxazole $(1.25 \mu \mathrm{g}+23.75 \mu \mathrm{g}))$, Vancomycin $(30 \mu \mathrm{g})$.

The diameter of the zone of inhibition for each antibiotic was measured and compared with that of the control organisms and interpreted as resistant, intermediate and sensitive according to Clinical Laboratory Standards Institute criteria. Klebsiella pneumoniae and E. coli that had ceftazidime zones $\leq 22 \mathrm{~mm}$ were tested for ESBL using ceftazidime discs and double-disk synergy (DDS) method. The antibiotics used were; ceftazidime $(30 \mu \mathrm{g})$ and ceftazidime-clavulanic acid. These antibiotics were placed at a distance of $30 \mathrm{~mm}$ from each other. The plates were incubated overnight at $37^{\circ} \mathrm{C}$. An increase of at least $5 \mathrm{~mm}$ in the zone diameter for ceftazidime-clavulanic acid versus the zone diameter with ceftazidime tested alone was used to confirm the presence of ESBLs as recommended by the Clinical and Laboratory Standards Institute. Klebsiella pneumoniae 700603 was used as an ESBL-positive reference strain while Escherichia coli ATCC 25922 was used as an ESBL-negative reference strain [13].

\subsection{Data Management and Analysis}

The raw data was entered into excel spreadsheets and later imported to Stata (version 12) software for statistical analysis. Descriptive statistics of demographic and clinical variables were summarized and presented as a percentage, mean with respective standard deviation for continuous variables, and frequencies and percentages for categorical variables. Age was categorised into two categories i.e. $>50$ years and $\leq 50$ years because catheterised patients who are above 50 years have increased risk of developing CAUTI due to reduced body immunity [14] [15].

Different species of bacteria were sorted out and proportions of each isolated bacterium were compared to assess the most prevalent species involved in CAUTIs. Chi square test was used to compare CAUTI cases according to age group, Gender, ward where the catheter was inserted, catheter days in insitu. Isolated bacteria were compared according to age group, Gender, ward where the catheter was inserted, catheter days in situ. Evaluations were carried out at $95 \%$ confidence level and $\mathrm{P}<0.05$ was considered statistically significant. Results were presented in form of tables and charts.

\subsection{Ethical Consideration}

Ethical clearance was granted by Mbarara University Research and Ethical Committee and administrative clearance was obtained from Kabale Regional Referral Hospital director. Only participants who consented were included in the study. All study results were shared with the clinicians to aid in management of 
patients.

\section{Results}

\section{Study Flow}

One hundred and sixty-nine patients were screened for eligibility to participate in this study in the month of April and May 2019.

150 were enrolled while 29 were excluded in the study as shown in Figure 1 below.

150 study participants were enrolled in the study. The majority of the study participants were female $(\mathrm{n}=111,74 \%)$. Most of the study patient participants were recruited from maternity ward $(n=77,51.3 \%)$. Most of study participants were 50 years and below $(\mathrm{n}=127,84.7 \%)$. The mean age was 33 with standard deviation of 14 years as shown in Table 1.

Following the urine culture from 150 catheterized patients, urine from 23 (15.3\%) patients showed significant growth after 48 hours of catheterization indicating overall CAUTI incidence of $15.3 \%$ as shown in Table 2. The results show that there was a statistically significant difference in CAUTI incidence according to length of stay of catheters $\left(\chi^{2}(1)=13.7149, P=0.001\right)$. Patients with longer catheter days had higher cases of CAUTI $(n=16,30.2 \%)$ compared to patients with short catheter days $(n=7,7.2 \%)$ as shown in Table 3 .

Patients who were above 50 years had higher cases of CAUTI $(n=12,52.2 \%)$ compared to patients who were below 50 years and below $(n=11,8.7 \%)$ indicating statistical significant differences in the CAUTI incidence according to age category $\left(\chi^{2}(1)=28.4001, \mathrm{p}=0.001\right)$ as shown in Table 4 .

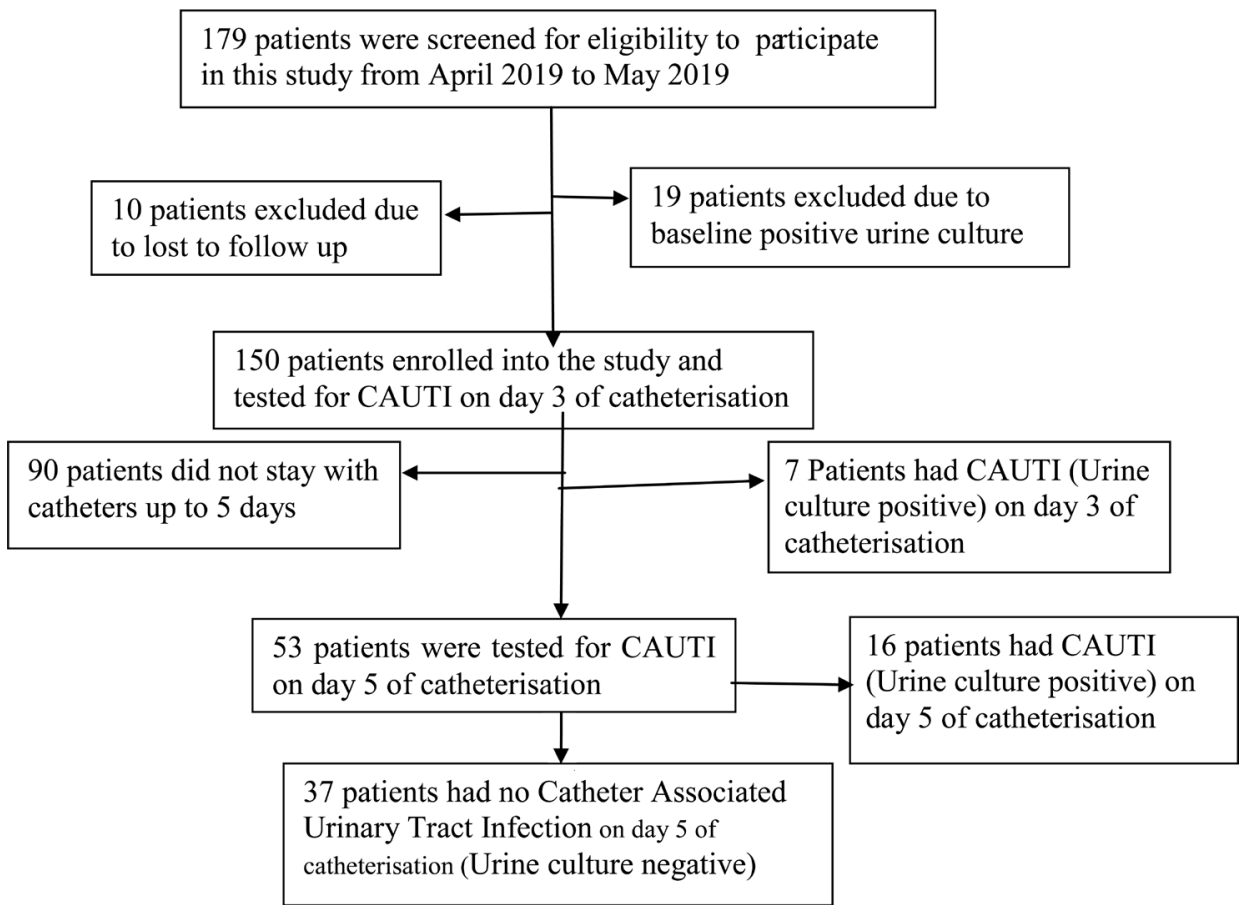

Figure 1. Study flow diagram. 
Table 1. Demographic characteristics of study participants $(\mathrm{N}=150)$.

\begin{tabular}{|c|c|c|c|}
\hline Characteristics & $N$ & $\%$ & Cumulative\% \\
\hline \multicolumn{4}{|l|}{ Gender } \\
\hline Male & 39 & 26 & 26.0 \\
\hline Female & 111 & 74 & 100 \\
\hline \multicolumn{4}{|l|}{ Age distribution } \\
\hline 1 to 20 & 22 & 14.7 & 14.7 \\
\hline 21 to 40 & 94 & 62.7 & 77.3 \\
\hline 41 to 60 & 24 & 16.0 & 93.3 \\
\hline \multirow[t]{2}{*}{61 to 80} & 10 & 6.7 & 100.0 \\
\hline & 150 & 100.0 & \\
\hline \multicolumn{4}{|l|}{ Age category } \\
\hline 50 years and below & 127 & 84.7 & 92.0 \\
\hline Above 50 years & 23 & 15.3 & 100 \\
\hline \multicolumn{4}{|l|}{ Ward } \\
\hline Emergency & 32 & 21.3 & 21.3 \\
\hline Surgical & 12 & 8.0 & 29.3 \\
\hline Medical & 15 & 10.0 & 44.0 \\
\hline Obstetrics and Gynecology & 14 & 9.3 & 53.3 \\
\hline \multirow[t]{2}{*}{ Maternity } & 77 & 51.3 & 100 \\
\hline & 150 & 100 & \\
\hline
\end{tabular}

Table 2. Overall CAUTI Incidence $\mathrm{N}=150$.

\begin{tabular}{cccc}
\hline CAUTI Incidence & N & $\%$ & Cumulative \% \\
\hline Negative & 127 & 84.7 & 84.7 \\
Positive & 23 & 15.3 & 100 \\
& 150 & 100 & \\
\hline
\end{tabular}

Table 3. Incidence of CAUTI according to length of stay of catheter.

\begin{tabular}{cccc}
\hline Catheter days & Before 3 Days & 3 - 5Days & Total \\
\hline CAUTI Incidence & & & \\
Positive n (\%) & $7(7.2)$ & $16(30.2)$ & $23(15.3)$ \\
Negative n (\%) & $90(92.8)$ & $37(69.8)$ & $127(84.7)$ \\
Total & $97(100)$ & $53(100)$ & $150(100)$ \\
Chi square value & & $\chi^{2}(1)=13.7149, \mathrm{P}=0.001$. & \\
\hline
\end{tabular}

From 23 bacterial isolates; Gram-negative bacteria 20 (87\%) were dominating as compared to Gram positive bacteria isolates $3(13 \%)$. The highest bacterial 
isolates were Escherichia coli 12 (52\%), followed by the Klebsiella pneumoniae 6 (26\%), Staphylococcus aureus 3 (13\%) and Pseudomonas spp. 2 (9\%) as shown in Figure 2.

Identified bacteria isolates (23) were tested for susceptibility to nine antibiotics. All Gram-negative isolates were sensitive to Imipenem 20 (100\%) while all $S$. aureus isolates (2) were $100 \%$ sensitive to Vancomycin and Cefoxitin. All isolates were $100 \%$ resistant to Cotrimoxazole. Most of the isolates were sensitive to Gentamicin 20 (82.6\%) and Ceftriaxone 16 (69.6\%). Sensitivity to Ciprofloxacin and Nitrofurantoin was $10(43.5 \%)$ and $9(39.1 \%)$ respectively. K. pneumoniae and Pseudomonas spp. isolates were $100 \%$ and $50 \%$ sensitive to gentamycin respectively. E. coli had the highest resistance to most antibiotics as shown in Table 5. Out of 20 Gram negative isolates, E. coli (3) and K. pneumoniae (3) isolates were resistant to Ceftazidime and they were tested for ESBL using ceftazidime $(30 \mu \mathrm{g})$ and ceftazidime-clavulanic acid double-disk synergy (DDS) method. Of the 6 tested isolates, 5 (83.3\%) were identified as ESBL-producing bacteria. K. pneumonia $3(60 \%)$ presented the highest percentage of ESBLs as compared to E. coli 2 (40\%) as shown in Figure 3.

Table 4. Incidence of CAUTI according to the age group of participants.

\begin{tabular}{cccc}
\hline & Age $\mathbf{5 0}$ years and below & Above $\mathbf{5 0}$ years & Total \\
\hline CAUTI Incidence & & & \\
Positive $\mathrm{n}(\%)$ & $11(8.7)$ & $12(52.2)$ & $23(15.3)$ \\
Negative $\mathrm{n}(\%)$ & $116(91.3)$ & $11(47.8)$ & $127(84.7)$ \\
Total & $127(100)$ & $23(100)$ & $150(100)$ \\
Chi square value & & $\chi^{2}(1)=28.4001, p=0.001$. & \\
\hline
\end{tabular}

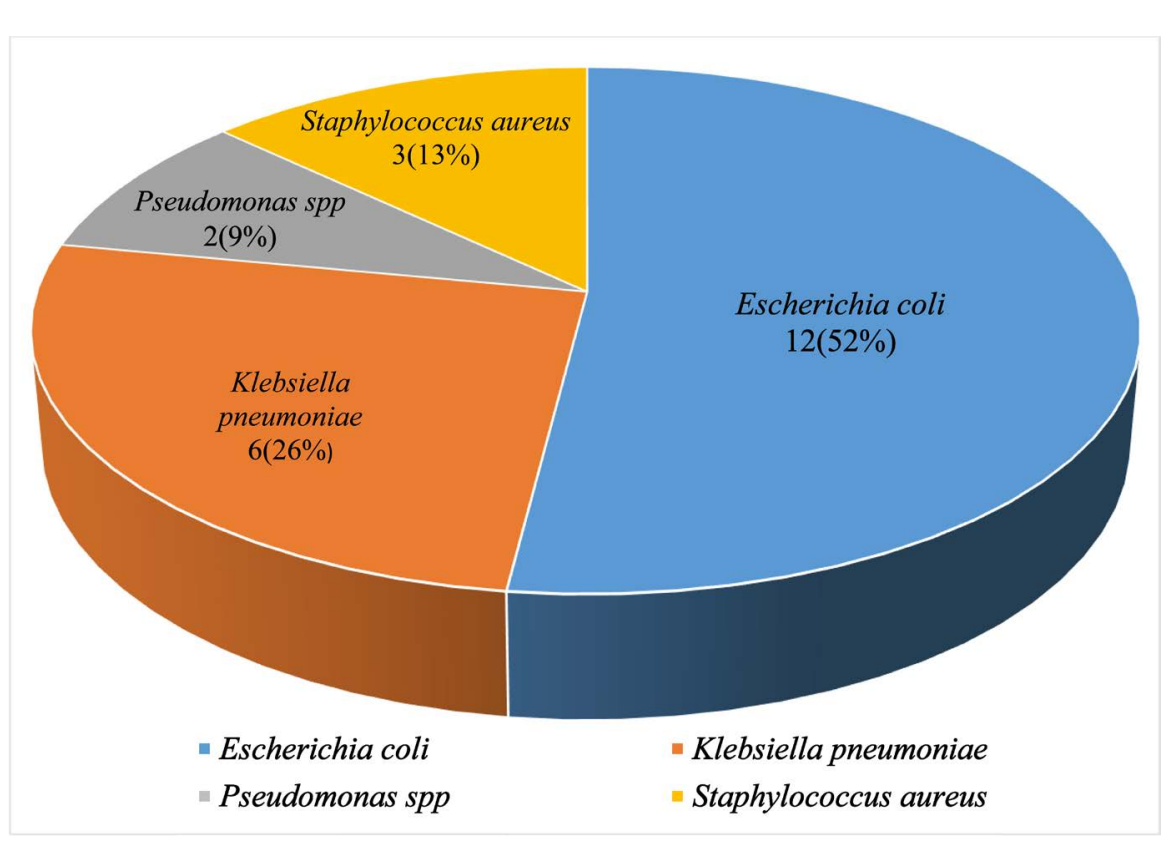

Figure 2. Bacteria isolates from the study participants $(n=23)$. 


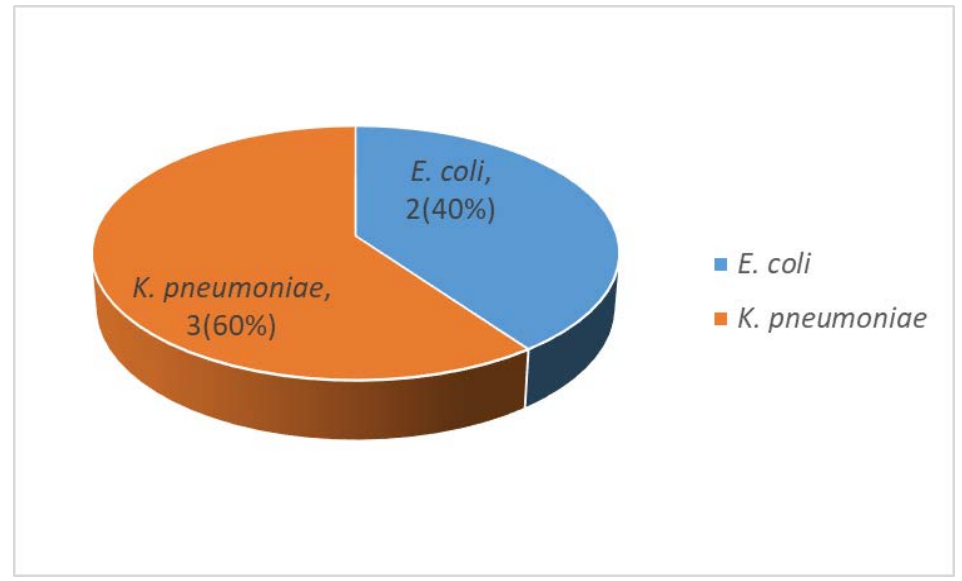

Figure 3. Gram-negative ESBL producing Bacteria $(n=5)$.

Table 5. Susceptibility patterns of bacteria to the different antibiotics.

\begin{tabular}{|c|c|c|c|c|c|c|}
\hline Antibiotic & Pattern & $\begin{array}{l}\text { E. coli } \\
\mathrm{n}=12\end{array}$ & $\begin{array}{c}K \cdot \text { pneumoniae } \\
\mathrm{n}=6\end{array}$ & $\begin{array}{c}\text { Pseudomonas spp. } \\
\mathrm{n}=2\end{array}$ & $\begin{array}{c}\text { S. aureus } \\
\mathrm{n}=3\end{array}$ & $\begin{array}{l}\text { Total } \\
\text { n (\%) }\end{array}$ \\
\hline \multirow{3}{*}{ Gentamycin } & $S$ & $10(83.3)$ & $6(100)$ & $1(50)$ & $2(66.7)$ & $20(82.6)$ \\
\hline & I & & & & & \\
\hline & $\mathrm{R}$ & $2(16.7)$ & $0(00)$ & $1(50$ & $1(33.3)$ & $4(17.4)$ \\
\hline \multirow{3}{*}{ Ceftriaxone } & S & $9(75)$ & $4(66.7)$ & $1(50)$ & $2(66.7)$ & $16(69.6)$ \\
\hline & I & & & & & \\
\hline & $\mathrm{R}$ & $3(25)$ & $2(33.3)$ & $1(50)$ & $1(33.3)$ & $7(30.4)$ \\
\hline \multirow{3}{*}{ Ciprofloxacin } & S & $3(25)$ & $4(66.7)$ & $2(100)$ & $1(33.3)$ & $10(43.5)$ \\
\hline & I & & & & & \\
\hline & $\mathrm{R}$ & $9(75)$ & $2(33.3)$ & $0(00)$ & $2(66.7)$ & $13(56.5)$ \\
\hline \multirow{3}{*}{ Cotrimoxazole } & S & $00(00)$ & $00(00)$ & $00(00)$ & $00(00)$ & $00(00)$ \\
\hline & I & & & & & \\
\hline & $\mathrm{R}$ & $12(100)$ & $6(100)$ & $2(100)$ & $3(100)$ & $23(100)$ \\
\hline \multirow{3}{*}{ Nitrofurantoin } & S & $6(50)$ & $1(16.7)$ & $1(50)$ & $1(33.3)$ & $9(39.1)$ \\
\hline & I & & & & & \\
\hline & $\mathrm{R}$ & $6(50)$ & $5(83.3)$ & $1(50)$ & $2(66.7)$ & $16(60.9)$ \\
\hline \multirow{3}{*}{ Ceftazidime } & S & $9(75)$ & $3(50)$ & $2(100)$ & - & $14(70)$ \\
\hline & I & & & & & \\
\hline & $\mathrm{R}$ & $3(25)$ & $3(50)$ & $0(00)$ & & $6(30)$ \\
\hline \multirow{3}{*}{ Imipenem } & S & & & & & \\
\hline & I & $12(100)$ & $6(100)$ & $2(100)$ & - & $20(100)$ \\
\hline & $\mathrm{R}$ & & & & & \\
\hline \multirow{3}{*}{ Vancomycin } & S & & & & & \\
\hline & I & - & - & - & $3(100)$ & $3(100)$ \\
\hline & $\mathrm{R}$ & & & & & \\
\hline \multirow{3}{*}{ Cefoxitin } & S & & & & & \\
\hline & I & - & - & - & $3(100)$ & $3(100)$ \\
\hline & $\mathrm{R}$ & & & & & \\
\hline
\end{tabular}

$\mathrm{S}=$ Sensitive, $\mathrm{I}=$ Intermediate, $\mathrm{R}=$ Resistant. 


\section{Discussion of Results}

\subsection{Discussion}

Use of urinary catheters is a known risk for urinary tract infection. Unfortunately, there is a paucity of data exploring the magnitude of CAUTI in Uganda. In this prospective descriptive hospital-based study, we sought to determine the incidence, etiology and antibiotic susceptibility pattern among the uropathens causing catheter Associated urinary tract infections in admitted patients with indwelling catheters.

The Incidence of CAUTI was $15.3 \%$ which reflects a high risk of developing hospital acquired CAUTI in patients with indwelling urinary catheters at Kabale RRH. This study is in agreement with numerous studies [10] [14] [15] [16] [17] revealing CAUTI as the most common hospital acquired infection among admitted patients. These findings do not vary much from CAUTI incidence of $11 \%$ among postpartum women at Mbarara Regional Referral Hospital [18] and incidence of $23 \%$ in Benin [14].

The high incidence of CAUTI at KRRH may be attributed to poor hygiene practices in relation to catheter handling, placement and maintenance. In addition previous studies have documented poor surveillance of hospital acquired infections, neglect of patient safety and health care quality as contributors to the high incidences of hospital acquired infections like CAUTI [19]. In a related study done in Zambia, a higher incidence of CAUTI (35\%) was reported [6], however, the duration of catherisation was longer (28days) implying that the longer a catheter stays in-situ, the more likely it is to lead to an infection within the urinary tract as reported in other studies [10]. This and other studies were done in Developing Countries document a higher incidence of CAUTI than the case in Developed Countries

Gardner et al., (2014) reported a CAUTI incidence of $0.9 \%$ in Australia as opposed to $15.3 \%$ in the current study. This finding is a reflective of the role of hygiene in preventing CAUTI. The findings of the current study indicated that CAUTI rates are higher in patients whose urethral catheters have stayed long compared to patients who had a short duration of catheterization $(30.2 \%$ vs $7.2 \%, \mathrm{p}=0.001)$. This is congruent with previous findings [14] in Benin and Zambia. The longer the catheter stays in situ, the higher the probability of developing CAUTI. The risk of acquiring catheter-associated bacteriuria is five percent for each day the catheter is in situ from day 5, this increases to 100 percent when the urethral catheter is in place for four weeks [10]. The incidence was particularly higher in elderly patients which might be a result of a weakened immunity [14].

The predominance of Gram-negative bacilli more especially $E$. coli and $K$. pneumoniae is in agreement with the study done in Benin showing gram negative bacilli as the most common cause of hospital acquired CAUTI [14]. This may be due to colonization of perineum by enteric bacteria that gain access to the urinary tract during catheterization [20]. Gram negative bacteria that 
cause CAUTI have a number of virulence factors related to motility, adhesion, immune avoidance, biofilm formation and nutrient acquisition that facilitates colonization and survival within the urinary tract [21]. Gram positive bacteria rarely cause UTI, indeed even in this study, we were only able to isolate $S$. aureus a fact that can be attributed to possession of lipoteic acid in the cell wall. This facilitates adhesion to the mucosal epithelium such that the bacterium is not flushed during urination [22].

Resistance to antibiotics is a growing concern Worldwide. In this study, E. coli had the highest resistance to all antibiotics (Table 5). This was similar to what was observed at the Regional hospital in Mbarara. Such findings indicate the magnitude of antibiotic resistance within the Western region in Uganda and calls for concerted efforts from all stake holders [18].

Relatedly, all bacteria isolates showed resistance to antibiotics that are commonly prescribed to treat UTIs. This makes it hard for clinicians to have wide choices of antibiotics for prescription to catheterized patients with CAUTI. All Gram-negative isolates were sensitive to Imipenem (100\%) and Gentamicin (83\%), similar findings were reported in the study titled "Antimicrobial susceptibility profiles of Escherichia coli and Klebsiella pneumoniae isolated from outpatients in urban and rural districts of Uganda" were Imipenem was confirmed as the only sensitive antibiotic against gram negative bacteria [23]. Unfortunately, the prohibitive cost of Imipenem and the nephrotoxic effects of gentamycin continue to restrict their use.

The Sensitivity observed for Ceftriaxone (69.6\%), Ciprofloxacin (43.5\%) Nitrofurantoin (39.1) and Cotrimoxazole (0\%) is comparable to antibiotic susceptibility in Mbarara RRH that showed sensitivity to ceftriaxone (75\%), Ciprofloxacin (66.6\%) and Cotrimoxazole (0\%) [24]. This may be attributable to the indiscriminate use of these antibiotics in empirical treatment of almost all ailments as well as use of some of these drugs (Cotrimoxazole) for prophylaxis in HIV infected individuals; thus bacteria are almost always exposed to such drugs which have led to resistance. The incidence of ESBL producing gram-negative bacteria dominated by Klebsiella pneumoniae (60\%) and Escherichia coli (40\%) in this study is in agreement with study findings by Baguma et al., (2017) indicating Klebsiella pneumoniae and Escherichia coli as the most ESBL producing bacteria [11].

This may be attributed to the empirical use of third generation ceftriaxone in combination with other antibiotics like gentamicin in treatment of most bacterial infections and prophylaxis among catheterized and surgical patients. Moreover, previous studies have characterised bacteria colonizing the drainage bags of catheterized patients as a major source of multidrug resistance [10]. The increasing cases of ESBL producing bacteria is a challenge to patient care, which renders use of third generation cephalosporins null. In such patients alternative like Carbapenems have to be considered yet they are expensive and unaffordable in a Country where the gross domestic product (GDP) is low. 


\subsection{Conclusion}

The Incidence of CAUTI among patients with indwelling urinary catheters at Kabale Regional Referral Hospital is high (15.3\%) and it is mainly caused by E. coli and $K$. pneumoniae. These bacteria are resistant to most commonly used antibiotics. There is a need to put more emphasis on CAUTI prevention strategies and use culture and sensitivity tests before prescription of antibiotics.

\subsection{Study Limitations}

Despite taking all due precautions to collect urine sample aseptically, the possibility of contamination could not be completely eliminated. Catheterized patients who were on antibiotics before and during sample collection were included in the study and this might have affected culture and sensitivity results.

\section{Acknowledgements}

We acknowledge the support and contribution from the Staff in the following Institutions; Department of microbiology Mbarara University of science and Technology, Hospital administration and laboratory management of Kabale Regional Referral Hospital, Department of Medical laboratory sciences Kampala International University Western campus and all the study participants.

\section{Conflicts of Interest}

The authors declare no conflicts of interest regarding the publication of this paper.

\section{References}

[1] Tambyah, P.A. and Oon, J. (2012) Catheter-Associated Urinary Tract Infection. Current Opinion in Infectious Diseases, 25, 365-370. https://doi.org/10.1097/QCO.0b013e32835565cc

[2] Bhani, D., et al. (2017) Microbial Profile and Antimicrobial Susceptibility Pattern of Uropathogens Isolated from Catheter Associated Urinary Tract Infection (CAUTI). International Journal of Current Microbiology and Applied Sciences, 6, 2446-2453. https://doi.org/10.20546/ijcmas.2017.608.289

[3] Melaku, S., et al. (2012) Antibiogram of Nosocomial Urinary Tract Infections in Felege Hiwot Referral Hospital, Ethiopia. African Health Sciences, 12, 134-139. https://doi.org/10.4314/ahs.v12i2.9

[4] Nwankwo, I., Godwin, C. and Nwankwo, E. (2014) Bacterial Profile in Patients with Indwelling Urinary Catheters in Federal Medical Center, Umuahia, Abia State, Nigeria. Sky Journal of Microbiology Research, 2, 28-31.

[5] Vyawahare, C.R., et al. (2015) Occurrence of Catheter-Associated Urinary Tract Infection in Critical Care Units. Medical Journal of Dr. D. Y. Patil Vidyapeeth, 8, 585. https://doi.org/10.4103/0975-2870.164974

[6] Labib, M. and Spasojevic, N. (2013) Problem of Catheter Associated Urinary Tract Infections in Sub-Saharan Africa. In: Nelius, T., Ed., Recent Advances in the Field of Urinary Tract Infections, InTech, London, 1-9. https://doi.org/10.5772/55371

[7] Beyene, G. and Tsegaye, W. (2011) Bacterial Uropathogens in Urinary Tract Infection and Antibiotic Susceptibility Pattern in Jimma University Specialized Hospital, 
Southwest Ethiopia. Ethiopian Journal of Health Sciences, 21, 141-146. https://doi.org/10.4314/ejhs.v21i2.69055

[8] Darbyshire, D., et al. (2016) Surveying Patients about Their Experience with a Urinary Catheter. International Journal of Urological Nursing, 10, 14-20. https://doi.org/10.1111/ijun.12085

[9] Hollingsworth, J.M., et al. (2013) Determining the Noninfectious Complications of Indwelling Urethral Catheters: A Systematic Review and Meta-Analysis. Annals of Internal Medicine, 159, 401-410. https://doi.org/10.7326/0003-4819-159-6-201309170-00006

[10] Nicolle, L.E. (2014) Catheter Associated Urinary Tract Infections. Antimicrobial Resistance and Infection Control, 3, 23. https://doi.org/10.1186/2047-2994-3-23

[11] Andrew, B., Kagirita, A. and Bazira, J. (2017) Prevalence of Extended-Spectrum Beta-Lactamases-Producing Microorganisms in Patients Admitted at KRRH, Southwestern Uganda. International Journal of Microbiology, 2017, Article ID: 3183076. https://doi.org/10.1155/2017/3183076

[12] Hall, G.S. (2015) Bailey \& Scott's Diagnostic Microbiology. 13th Edition, American Society for Clinical Pathology, Chicago.

[13] Patel, J.B. (2017) Performance Standards for Antimicrobial Susceptibility Testing. Clinical and Laboratory Standards Institute, Wayne.

[14] Dougnon, T.V., et al. (2016) Catheter-Associated Urinary Tract Infections at a Hospital in Zinvie, Benin (West Africa). International Journal of Infection, 3, e34141. https://doi.org/10.17795/iji-34141

[15] Lo, E., et al. (2014) Strategies to Prevent Catheter-Associated Urinary Tract Infections in Acute Care Hospitals: 2014 Update. Infection Control \& Hospital Epidemiology, 35, S32-S47. https://doi.org/10.1017/S0195941700095382

[16] Gardner, A., et al. (2014) A Point Prevalence Cross-Sectional Study of Healthcare-Associated Urinary Tract Infections in Six Australian Hospitals. BMJ Open, 4, e005099. https://doi.org/10.1136/bmjopen-2014-005099

[17] Chitnis, A.S.,et al. (2012) Device-Associated Infection Rates, Device Utilization, and Antimicrobial Resistance in Long-Term Acute Care Hospitals Reporting to the National Healthcare Safely Network, 2010. Infection Control \& Hospital Epidemiology, 33, 993-1000. https://doi.org/10.1086/667745

[18] Bebell, L.M.,et al. (2017) Antimicrobial-Resistant Infections among Postpartum Women at a Ugandan Referral Hospital. PLoS ONE, 12, e0175456. https://doi.org/10.1371/journal.pone.0175456

[19] Murugan, K., Selvanayaki, K. and Al-Sohaibani, S. (2016) Urinary Catheter Indwelling Clinical Pathogen Biofilm Formation, Exopolysaccharide Characterization and Their Growth Influencing Parameters. Saudi Journal of Biological Sciences, 23, 150-159. https://doi.org/10.1016/j.sjbs.2015.04.016

[20] Flores-Mireles, A.L., et al. (2015) Urinary Tract Infections: Epidemiology, Mechanisms of Infection and Treatment Options. Nature Reviews Microbiology, 13, 269-284. https://doi.org/10.1038/nrmicro3432

[21] Khaledi, A., et al. (2018) Prevalence of Catheter-Associated Bacteriuria in Patients Who Received Short-Term Catheterization in the Northeast of Iran. Novelty in Biomedicine, 6, 79-84.

[22] Nicolle, L.E. (2012) Urinary Catheter-Associated Infections. Infectious Disease Clinics of North America, 26, 13-27. https://doi.org/10.1016/j.idc.2011.09.009

[23] Najjuka, C.F., et al. (2016) Antimicrobial Susceptibility Profiles of Escherichia coli 
and Klebsiella pneumoniae Isolated from Outpatients in Urban and Rural Districts of Uganda. BMC Research Notes, 9, 235. https://doi.org/10.1186/s13104-016-2049-8

[24] Ampaire, L., et al. (2015) Bacterial and Drug Susceptibility Profiles of Urinary Tract Infection in Diabetes Mellitus Patients at Mbarara Regional Referral Hospital, Uganda. British Microbiology Research Journal, 9, 1-5.

https://doi.org/10.9734/BMRJ/2015/17483

[25] Ramesh, A., et al. (2018) Microbiological Profile, Comorbidity, Incidence and Rate Analysis of Catheter Associated Urinary Tract Infections in Adult Intensive Care Unit. 